\section{Antimicrobial Stewardship Practices in Michigan Long-Term Care Facilities}

To the Editor-Antimicrobial resistance is a public health crisis. A major strategy to combat antimicrobial resistance in the recent US government's National Action Plan for Combating Antibiotic-Resistant Bacteria is the implementation of antimicrobial stewardship programs (ASPs). ${ }^{1}$ Development of ASPs in long-term care facilities (LTCF) has been limited despite the heavy use of antimicrobials and high prevalence of resistant organisms and Clostridium difficile infection in these settings. ${ }^{2-6}$

The US population continues to age, with an estimated $21 \%$ of the population by 2040 consisting of persons 65 years of age and older. ${ }^{7}$ There are currently 15,700 nursing homes in the United States, with an estimated 1.4 million residents. ${ }^{8}$ A reported $47 \%$ to $79 \%$ of LTCF residents receive antimicrobials each year. ${ }^{4}$ Given the call to implement ASPs across the continuum of care and limited data on antimicrobial stewardship practices in LTCF, we assessed these practices throughout Michigan.

The Michigan Department of Health and Human Services and a team of infectious disease physicians and pharmacists distributed a web-based survey to LTCF from August to September, 2014, through multiple listservs: Michigan Society for Infection Prevention and Control, Association for Professionals in Infection Control and Epidemiology-Great Lakes Chapter, Michigan Healthcare Associated Infections Prevention Advisory Committee, Michigan Chapter of the National Association Directors of Nursing Administration, and MPRO (formerly Michigan's healthcare quality improvement organization). Survey questions characterized current antimicrobial stewardship practices, educational and stewardshiprelated needs, and ways that our team could provide assistance. For this survey, an ASP was defined as coordinated interventions to promote judicious antimicrobial use.

Responses were received from 86 LTCF, and 45 (52\%) had greater than 100 licensed beds (median size, 111 beds). ASP policies and procedures were reported in 60 (75\%) of 80 LTCF. However, only 15 (23\%) of 66 facilities reported having a formal ASP with dedicated staffing. Of 41 responding LTCF, $23(56 \%)$ reported having a stewardship or similar committee that monitored antimicrobial utilization. Team members involved in monitoring the ASP or antimicrobial utilization included infection preventionists $(85 \%)$, medical directors (48\%), nurses $(48 \%)$, and pharmacists $(43 \%)$.

Thirty-eight respondents described which antimicrobials their policies and procedures addressed. These responses included all antimicrobials in 17 (45\%), antimicrobials based on resistance profiles in $16(42 \%)$, antimicrobials used in
C. difficile infection treatment in 14 (37\%), broad-spectrum antimicrobials in $11(29 \%)$, and high cost antimicrobials in $10(26 \%)$. Of 39 responding LTCF, strategies to improve antimicrobial prescribing included clinical review in $35(90 \%)$, antimicrobial de-escalation in 19 (49\%), formulary restriction in $14(36 \%)$, and intravenous to oral conversion (35\% [13/ 37]). Thirty-nine $(52 \%)$ of 75 respondents monitored or quantified antimicrobial consumption while a similar percentage $(58 \%$ [43/74]) reviewed antibiogram data. Of 39 LTCF, 29 (74\%) developed process improvements plans if high rates of inappropriate antimicrobial utilization were identified.

Obstacles for implementation of ASPs in 26 responding LTCF included lack of knowledge regarding ways to initiate a program in $14(54 \%)$, absence of a proposal in $13(50 \%)$, staffing constraints in $2(8 \%)$, and lack of prioritization in $2(8 \%)$. Overall, LTCFs were interested in educational opportunities surrounding antimicrobial stewardship. Of the 47 facilities interested in training, $34(72 \%)$ desired training for clinical guideline development, $32(68 \%)$ sought education on antimicrobial de-escalation, 29 (62\%) requested assistance measuring outcomes, 27 (57\%) desired training on antimicrobial dose optimization, 27 (57\%) requested assistance developing buy-in from staff, and 17 (36\%) desired support from administration.

More than half of responding LTCF had greater than 100 licensed beds, and most (75\% [60/80]) reported having ASP policies and procedures. However, few LTCF (23\% [15/66]) had a formal ASP with dedicated staffing. Our results are consistent with what others have reported. LTCF have been slower to adopt stewardship strategies and measures given lack of electronic resources and funding as well as limited evidenced-based stewardship strategies specific to LTCF. ${ }^{2,3,6,9}$ The long-term care environment is complex. There is often a lack of on-site diagnostic testing and many prescriptions are called by telephone order, frequently by on-call providers, without a preceding physical examination. ${ }^{10}$ There is a lack of infectious diseases expertise, making concurrent review and adjustment of antimicrobial therapy challenging.

In contrast to acute care facilities, the team members most frequently involved in the ASP or monitoring antimicrobial utilization in LTCF were infection preventionists, medical directors, and nurses. LTCF have variable models of physician presence with the largest proportion of in-person staffing consisting of nursing. ${ }^{4}$ As noted by our responses, there is an urgent need for antimicrobial stewardship education in LTCF because there is a lack of real-time infectious diseases physician and pharmacist expertise.

Our study has some limitations. Michigan has roughly 440 LTCFs and skilled nursing facilities. It proved difficult to quantify the exact number and types of facilities reached by the survey. Though we attempted to cover multiple groups and disciplines, some facilities may not be a part of the listservs 
used for distribution. As a crude estimate, our response rate was 20\% (86/440); however, this could not be confirmed. Second, facilities with an ASP may be more likely to respond, leading to selection bias.

Antimicrobials are commonly prescribed in LTCF. The extensive use of antimicrobials results in the risk of not only adverse drug reactions, but also the promotion of antimicrobial resistance and $C$. difficile infection. Antimicrobial stewardship is relatively uncommon in surveyed LTCF across Michigan. Education and training pertaining to antimicrobial stewardship are sorely needed for LTCF. Development of ASPs tailored to the needs and resources of LTCFs will be essential to prevent further emergence of antimicrobial resistance across the continuum of care.

\section{ACKNOW LEDGMENTS}

Financial support. None reported.

Potential conflicts of interest. All authors report no conflicts relevant to this article.

\section{Anurag N. Malani, MD; ${ }^{1,2}$ Brenda M. Brennan, MSPH; ${ }^{3}$ Curtis D. Collins, PharmD, MS; ${ }^{4}$ Jennie Finks, DVM, MVPH; ${ }^{3}$ Jason M. Pogue, PharmD; $;^{5,6}$ Keith S. Kaye, MD, MPH ${ }^{6,7}$}

Affiliations: 1. Division of Infectious Diseases, Department of Internal Medicine, St. Joseph Mercy Hospital, Ann Arbor, Michigan; 2. Department of Infection Prevention and Control, St. Joseph Mercy Hospital, Ann Arbor, Michigan; 3. Michigan Department of Health and Human Services, Lansing, Michigan; 4. Department of Pharmacy, St. Joseph Mercy Hospital, Ann Arbor, Michigan; 5. Division of Pharmacy Services, Sinai-Grace Hospital, Detroit, Michigan; 6. Wayne State University, School of Medicine, Detroit, Michigan; 7. Detroit Medical Center, Detroit, Michigan.

Address correspondence to Anurag N. Malani, MD, St. Joseph Mercy Hospital, 5333 McAuley Dr, Ste 6109, Ypsilanti, MI 48197 (anurag.malani@ stjoeshealth.org).

Infect. Control Hosp. Epidemiol. 2016;37(2):236-237

(c) 2015 by The Society for Healthcare Epidemiology of America. All rights reserved. 0899-823X/2016/3702-0022. DOI: 10.1017/ice.2015.286

\section{REFERENCES}

1. National action plan for combating antibiotic-resistant bacteria. The White House website. www.whitehouse.gov. Published March 2015. Accessed July 1, 2015.

2. Van Schooneveld T, Miller H, Sayles H, Watkins K, Smith PW. Survey of antimicrobial stewardship practices in Nebraska long-term care facilities. Infect Control Hosp Epidemiol 2011;32:732-734.

3. Jump RL, Olds DM, Seifi $\mathrm{N}$, et al. Effective antimicrobial stewardship in a long-term care facility through an infectious disease consultation service: keeping a LID on antibiotic use. Infect Control Hosp Epidemiol 2012;33:1185-1192.

4. Rhee S, Stone N. Antimicrobial stewardship in long-term care facilities. Infect Dis Clin N Am 2014;28:237-246.

5. Smith PW, Bennett G, Bradley S, et al. SHEA/APIC guideline: infection prevention and control in the long-term care facility. Am J Infect Control 2008;36:504-535.
6. Smith PW, Watkins K, Miller H, VanSchooneveld T. Antibiotic stewardship programs in long-term care facilities. Ann LongTerm Care Clin Care Aging 2011;19:20-25.

7. Administration on Aging. A profile of older Americans. Administration on Aging website. http://www.aoa.gov/Aging_Statistics/ Profile/2012/docs/2012profile.pdf. Published 2012. Accessed July 1, 2015.

8. FastStats: nursing home care. Centers for Disease Control and Prevention website. http://www.cdc.gov/nchs/fastats/nursinghome-care.htm. Accessed July 1, 2015.

9. Dellit TH, Owens RC, McGowan JE, et al. Infectious Diseases Society of America and the Society for Healthcare Epidemiology of America guidelines for developing an institutional program to enhance antimicrobial stewardship. Clin Infect Dis 2007;44: 159-177.

10. Daneman N, Gruneir A, Bronskill S, et al. Prolonged antibiotic treatment in long-term care. JAMA Intern Med 2013;173:673-682.

\section{Surveillance Systems for Nosocomial Infections: Methods and Challenges}

To the Editor-Nosocomial infections (NIs), also known as healthcare-associated infections and hospital-acquired infections, with a pooled prevalence of $10.1 \%$, are considered to be serious public health problems around the world. ${ }^{1}$ The NI-related burden is unknown because of lack reliable data, lack of surveillance systems and the complexity of corresponding NI outcomes. ${ }^{2}$

Healthcare systems use different approaches to monitoring NIs. ${ }^{3-7}$ Generally, surveillance methods are categorized under the umbrellas of active, passive, and sentinel surveillance methods. ${ }^{8}$ At present, hospitals implement passive surveillance approaches because of feasibility and low cost. However, the quality of this methodology is in question; underreporting and lack of timeliness are the main challenges. In contrast to the passive approach of NI surveillance systems, active ones do not face the challenge of real-time detection of hospital-acquired infection and provide high-quality data on the trends and burdens of NIs. This approach requires extensive resources.

Lessons learned from the implementation of passive approaches to NI surveillance, especially in low- and middleincome countries, have revealed the necessity of applying other methods. Implementation of integrated sentinel surveillance methods using active approaches at selected hospitals and healthcare facilities provides reliable data about the epidemiological profiles of hospital-acquired infections with limited resources. Selecting representative hospitals can contribute to an appropriate understanding of NI-related burden.

\section{ACKNOWLEDGMENTS}

Financial support: No financial support was provided relevant to this article. 